\title{
HYPERSPECTRAL PANORAMIC IMAGING
}

\author{
M. Müller-Rowold ${ }^{\mathrm{a}}$, R. Reulke ${ }^{\mathrm{b} *}$ \\ a jember GmbH, Germany - malte.mueller-rowold@jember.de \\ ${ }^{\mathrm{b}}$ HU-Berlin, Germany - reulke@informatik.hu-berlin.de
}

WG I/1

KEY WORDS: Hyperspectral camera, hyperspectral imaging, Fabry-Perot spectral filters

\begin{abstract}
:
Hyperspectral instruments are designed for the characterisation of planetary surfaces, oceans and the atmosphere. At the moment there are a number of aircraft systems and planned space missions. Examples for this are the hyperspectral missions for Earth remote sensing (EnMAP) and also for deep space and planetary missions (Mercury mission Bepi Colombo).

There are basically two options for a hyperspectral system: Snapshot systems and scanning systems. This paper investigates a scanning hyperspectral push-broom systems. In most systems the input aperture is a long slit whose image is dispersed across a 2-D detector array, so that all points along a line in the scene are sampled simultaneously. To fill out the spatial dimension orthogonal to the slit, the scene is scanned across the entrance aperture. An ideal low cost hyperspectral scanning device analogue to push broom scanner is a 2D-detector with variable spectral filters, each filter being arranged perpendicular to the direction of flight.

The biggest challenge is the mapping of the images of the individual spectral channels to each other (co-registration). The solution of the problem is the prerequisite for the use of this kind of hyperspectral cameras e.g. on board of an aircraft. Therefore, an investigation should focus on the procedure of data acquisition, correction and registration. In addition, an example showing the advantages of a possible application is explained.
\end{abstract}

\section{INTRODUCTION}

High resolution imaging spectrometry or hyperspectral imaging (HSI) requires sophisticated solutions both for the detector and its operation as well as for the hardware and mechanics. In particular, the spectrograph with grating or prism is expensive. Detector and grating ensure that the hyperspectral imager can only operate over a limited spectral range (e.g. VIS/NIR or SWIR). Alternative approaches are optical band pass filter. In this paper a scanning hyperspectral push-broom systems was investigated.

An important characteristic for the spectral resolution of a camera system besides its number of spectral channels is the full width at half maximum (FWHM) of each of its bands. Multispectral camera systems capture images with only a few broad bands with a few hundred nm. Hyperspectral systems, on the other hand reach a much higher spectral resolution through many narrow bands (100-200 bands with a FWHM of 5-10 nm).

The measured pixel value corresponds with the reflected portions of light in this spectral range by the captured scene and describes the signatures of its objects which can be used for further analysis and classification.

Applications for hyperspectral systems include precision farming, environmental monitoring, quality control in the food and medical industry, biotechnology as well as security and defence.

Most systems use complex bulky optical systems with gratings or prisms for the decomposition of the incident light. An alternative and compact system was introduced by the Belgian company Imec by monolithically integrating Fabry-Perot spectral filters on top of the pixels of a commercial CMOS-sensor at wafer-level

\footnotetext{
${ }^{*}$ Corresponding author
}

(Imec, Jerome Baron, 2017). As a first test case for evaluating the system a setup for capturing a $360^{\circ}$ hyperspectral panorama was developed and is presented here.

The paper is organised as follows. First, a brief overview of the theory is given. Then the measurement setup, spectral property verification, flat-field correction and acquisition settings are described. In the next chapter the raw data evaluation and processing is described. Finally, a brief overview of the results is given. The paper comes up with conclusions and an outlook.

\section{PANORAMIC IMAGING}

\subsection{Basics}

The result of a hyperspectral data acquisition is a data cube. The used hyperspectral systems requires a linear movement to obtain a complete image. In (Karaca et al., 2016) a system capturing a stereo hyperspectral panorama was introduced using relatively small spectrometers and scanning spatially. The here used approach using filters directly applied to the detector surface is smaller and achieves an improved spatial and spectral resolution. In contrast to the used system developed by Imec a spatio-spectral scan procedure has to be performed. This is due to the filters being applied in a line-scan layout onto a matrix detector. Thus each spectral line acquires a different spatial stripe (see Figure 1 .

The model for this setup is based on a rotating multi-sensor-line camera as in Figure 2, where each sensor column records a separate spectral channel. Therefore the camera is tilted $90^{\circ}$ and is then incrementally rotated around an axis. If the projection centre of the camera is not exactly located on the rotation centre, there will be an off-axis distance $R$ and for each angle increment the cameras position is moving on a circle around the axis resulting 


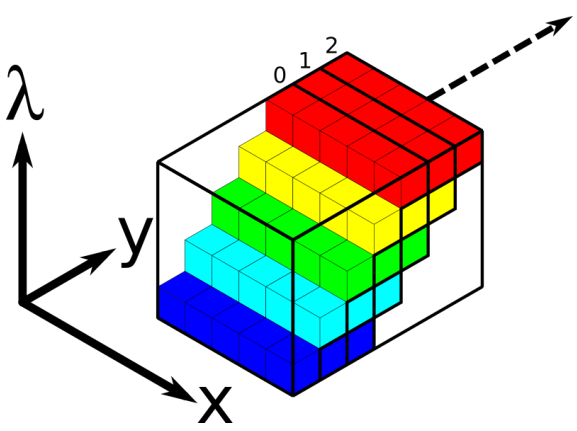

Figure 1. Spectral scanning method

in parallax effects between the captured frames. After scanning each spectral column can be stitched together to build a whole image cylinder.(Huang et al., 2008 Huang et al., 2006) The required incremental step for a single centre panorama can be calculated from the focal length $f$ and the pixel size $\tau$ using the equation of the IFOV.

$$
I F O V=2 \cdot \tan ^{-1}\left(\frac{1}{2} \frac{\tau}{f}\right)
$$

The spectral columns $i$ are then characterised by the angle $\omega$ between the cylinder normal at the principal point where the centre column $i_{c}$ is located and the ray passing through the centre of the column $i$ from the centre of projection.

$$
\omega=\tan ^{-1}\left(\frac{\left(i-i_{\mathcal{C}}\right) \tau}{f}\right)
$$

In case of a single centre panorama the spectral columns capture all the same parallax-free panorama which differs only by different scales and translations.(Huang et al., 2008. Huang et al., 2006)

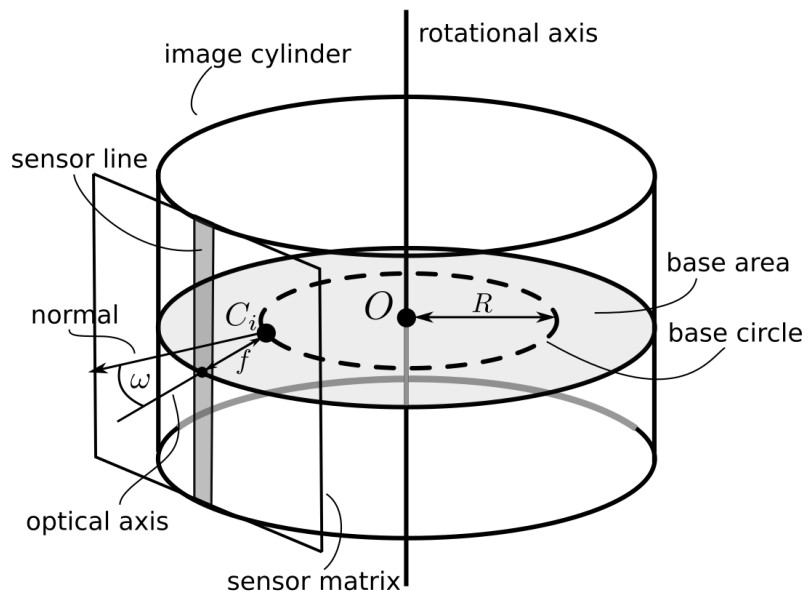

Figure 2. Model of a rotating matrix-sensor or multi-sensor-line

\subsection{Measurement setup}

A construction of a rotating line camera is seen in Figure 4 consisting of three basic components:

\section{- Camera head}

- Optical bench
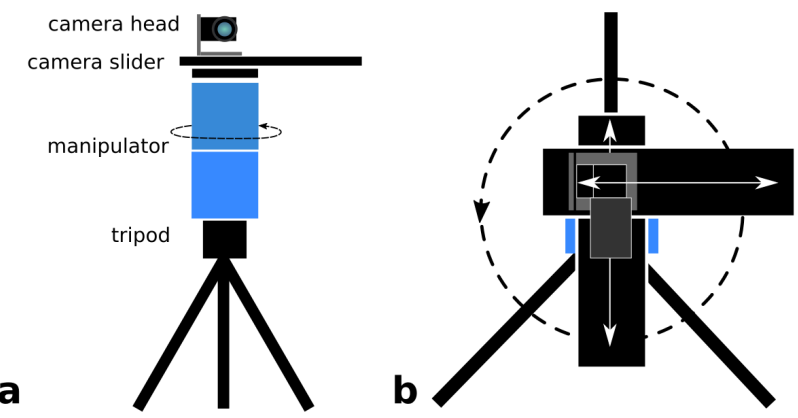

Figure 3. Measurement setup sketch, a) side-view, b) top-view

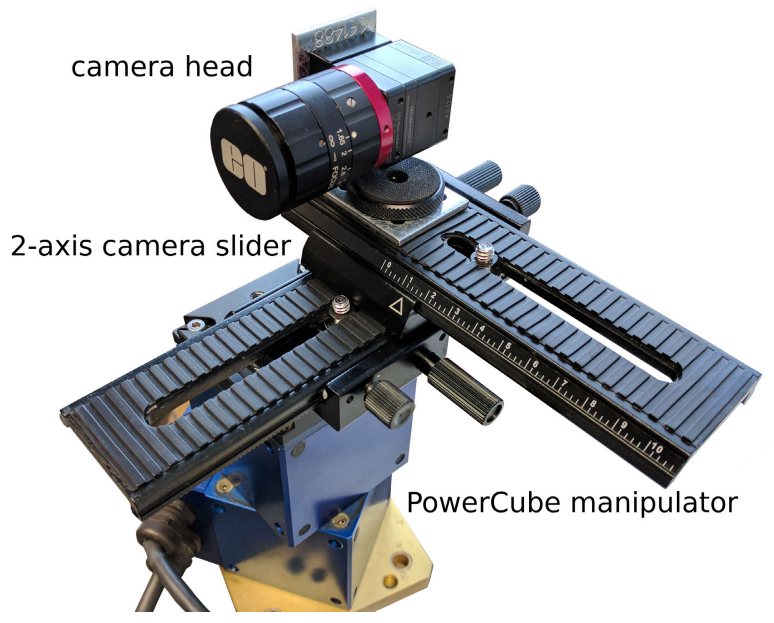

Figure 4. Measurement setup

- Turntable / drive

The camera head consists of the hyperspectral detector manufacture by the company Imec. Its filters are applied on an infrared enhanced CMV2000 sensor in a line-scan layout. Result of spectral calibration are presented by (Spiess and Reulke, 2017). Each spectral channel has the size of 2048 pixels $\times 8$ lines. The sensor is integrated into the compact industrial USB3 camera $M Q 022 H G-I M-L S 100-N I R$ of the family $x i Q$ provided by the company Ximea. It includes a fixed focal length lens from Edmund optics with $f=35 \mathrm{~mm}$ and a band-pass filter blocking wavelengths below $600 \mathrm{~nm}$ and above $975 \mathrm{~nm}$.
resolution
pixel size
sensor size
bit depth
framerate

$$
\begin{gathered}
2048 \times 1088 \mathrm{px}(2.2 \mathrm{MP}) \\
5.5 \mu \mathrm{m} \times 5.5 \mu \mathrm{m} \\
2 / 3 \mathrm{in} \\
10 \mathrm{bit},(12 \mathrm{bit}) \\
340 \text { FPS (10 bit), } 70 \text { FPS (12 bit) }
\end{gathered}
$$

Table 1. Sensor characteristics of the CMV2000

Fabry-Prot interference filters transmit only wavelengths of light satisfying its resonance condition which includes second order harmonics:

$$
k \lambda=2 n_{G} L \cos \theta
$$

The medium between the two partially highly reflecting surfaces of the filter defines the refractive index $n_{G}$. Their distance is $L$ and the angle of incident light is $\theta$. 
A bracket attaches the camera head onto the optical bench achieving the tilt of the camera. Two camera sliders are used as optical bench for fine tuning the position of the camera towards the centre of rotation in two axis and thus the distance $R$.

The rotation is achieved by a PowerCube module from Schunk acting as closed-loop manipulator. It features a geared brushless DC motor which is PID controlled using optical encoder feedback. The PowerCube is commanded via a serial RS-232 interface and an USB-adapter.

\subsection{Filter verification}

The filters of the detector are specified to lie in the range from 600 to $1000 \mathrm{~nm}$ with a step of around $4 \mathrm{~nm}$ between two adjacent ones. The real characteristics of the filters are calibrated and the resulting data is provided by Imec. From a total of 128 filters only 104 lie within their tolerance reaching from spectral pixel index 124 to 955 . The raw data taken from the calibration data shows the need for the band-pass filter supplied with the camera as the filters are hardly distinguishable here. Figure 5 shows the validation results of the calibration data using a monochromator setup with the complete camera including the band-pass filter. The measured data correlates to the provided data only differing in the band-passed range and higher FWHM values which are caused by the aperture of the camera. As stated in (Tack et al., 2012) widening the aperture has an impact on the transmission efficiency and spectral resolution of the filters caused by the differing incident light angles compared to the calibration by Imec, where collimated light was used.(Tack et al., 2012) The measured data shows that the second order harmonics are causing the filters to have multiple peaks in their transmitted wavelengths which can result in a wrong spectrum captured.

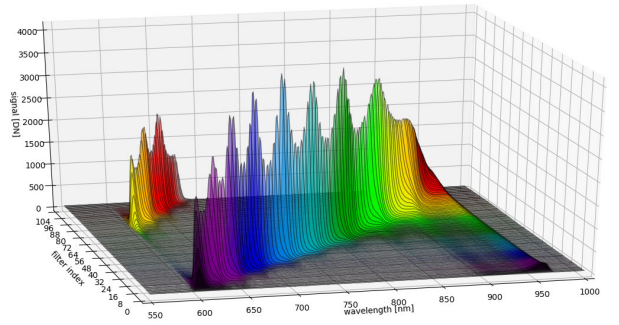

Figure 5. Spectral measurement of the system with a monochromator

\subsection{DSNU and PRNU correction}

The measured digital signals have to be corrected by pixel dependent dark signal (DSNU - dark signal non-uniformity) and individual pixel response (PRNU - pixel response non-unifomity). DSNU depends on the exposure time and the temperature of the detector.

The measurement of the dark signal non-uniformity (DSNU) is done with an exposure series. The values for each pixel are averaged to reduce the effect of random noise. From the measured data the Offset value $e_{D O}^{-}$and the exposure time dependent $e_{D C}^{-}$ are calculated by linearly approximating the measurement. The correction is done by subtracting the interpolated dark signal value from each pixel.

The photo-response non-uniformity is measured using a time stable homogeneous integrating sphere as an uniformly illuminating light source to provide a calibrated irradiance for the detector. The correction of the DSNU is done before. The detector is only operated in the linear range outside of the saturation. Assuming a linear relation between the irradiance and the response of the detector, a frame with 50 to $70 \%$ saturation is captured. The multiplicative correction value for each pixel is calculated by dividing the mean value of all detector pixels by the individual pixel value. The PRNU depends from the wavelengths of the incident light. Therefore each filter of the hyperspectral detector is calibrated and corrected individually. (Mansouri et al., 2005) Figure 6 shows the measured and calculated correction coefficients for an aperture of $f / 4$.

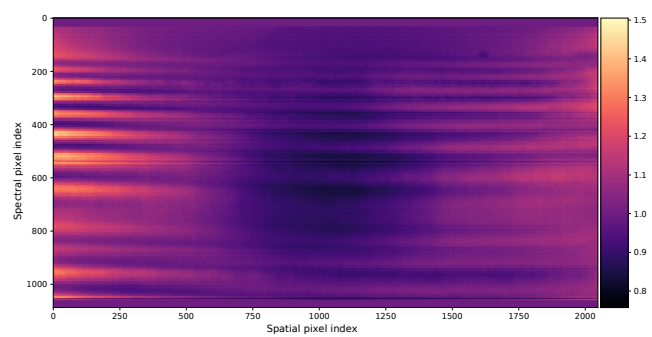

Figure 6. PRNU correction coefficients for aperture $\mathrm{f} / 4$

\subsection{Procedure for centring the camera}

To prevent parallax errors in the captured panorama it is crucial that there is no off-axis distance $R$ and thus the setup is singlecentred as mentioned above. The projection centre of the camera lies within the centre of the entrance pupil of the lens. To achieve coincident positions of the rotation centre and the central entrance pupil, a grid test procedure is carried out.(Hamblenne, 2004)

1. The correct position of the camera sliders are visually estimated and set up. As a result the lens is roughly centred on the rotation axis.

2. Then a grid is placed in the foreground and a scene with a similar structure is observed in the background.

3. The parallax error is evaluated by the relative orientation between grid and background when observing the current angle and the angle, where the leftmost image segment has moved to the rightmost image segment. Then the camera sliders are adjusted until there is no parallax error left.

Figure 7 illustrates this procedure. The grid is highlighted green while the building structure in the background is left monochrome. In A the image section is seen leftmost while after rotation in $\mathrm{B}$ it is seen in the rightmost area of the sensor. There is still a deviation in the orientation between fore- and background and the sliders have to be adjusted.

\subsection{Acquisition methods}

In order to capture the image columns on the exact positions defined by the step angle, the rotation of the PowerCube has to be synchronised with the image acquisition of the camera. If not, there should be at least an overlap between two frames. Missing frames will result in gaps in the panorama, which will occur in different positions in the spectral channels. The certain method to realise this is by performing both actions sequentially. This 


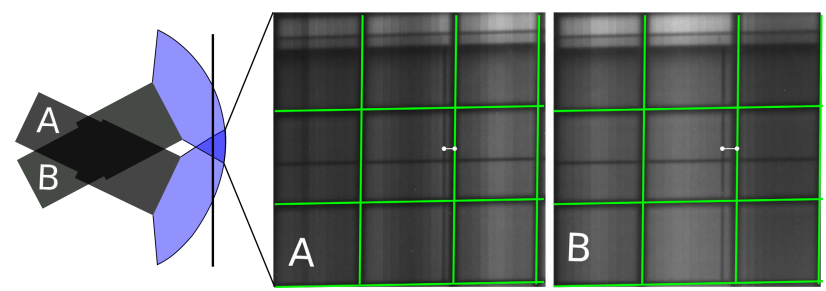

Figure 7. Grid test for aligning the entrance pupil to the rotational centre

means that the camera is idle during rotation which is slowing down the acquisition significantly. If the manipulator takes too long for finding the correct position the acquisition time is drastically increased. This also requires the manipulator to be precise and not overshoot the step positions.

The maximum step angle required for the camera is calculated by the column widths and the IFOV:

$$
8 \cdot I F O V=16 \cdot \tan ^{-1}\left(\frac{5.5 \mu \mathrm{m}}{2 \cdot 35 \mathrm{~mm}}\right) \approx 0.072^{\circ}
$$

For a full $360^{\circ}$ cylinder with no overlap 5000 frames have to be captured. For $1 \mathrm{~s}$ positioning time the whole acquisition takes up more than one hour with lots of scene changes causing differences between the channels. Another method is performing both actions parallel. Then the frame rate of the camera and the constant rotation of the PowerCube have to be set to achieve the desired step angle. This results in motion blur for the acquired frames which can be limited by setting the rotation speed and exposure time accordingly. The final panorama was acquired using constant speed of $0.36^{\circ} / \mathrm{s}$, an exposure time of $25 \mathrm{~ms}$ and a frame rate of $10 \mathrm{fps}$ resulting in 1 pixel motion blur and an 4 pixel overlap between adjacent steps. The image acquisition for the full $360^{\circ}$ panorama took around 17 minutes.

\section{DATA PROCESSING}

\subsection{Stitching}

The result of the image acquisition is a batch of 10000 raw images with a resolution of $2048 \times 1088 \mathrm{px}$. After DSNU and PRNU correction their spectral channels (4 lines) are stitched together to the 104 final hyperspectral cube with a resolution of $2048 \times 40000 \mathrm{px} \times 104$ channels each.
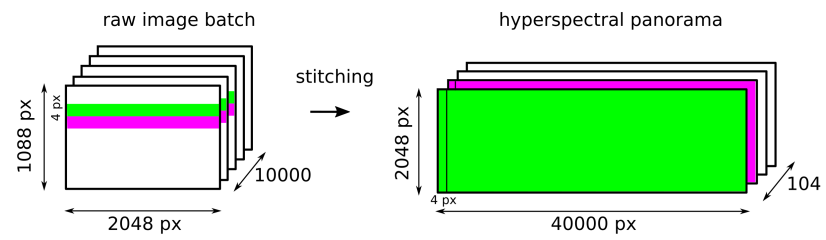

Figure 8. Stitching process

In case of an uneven or imprecise step angle the lines have to be stitched with sub-pixel accuracy. As stated above the spectral channels each build up a complete panorama which differ in translation and scale which has to be corrected by aligning them.

\subsection{Spectral channel alignment}

Aligning the spectral channels is corresponds to their registration and transformation under the assumption of a specific motion model. A reference channel is chosen while for the rest a transform is estimated to match the target. In case of the rotating sensor matrix at least a similarity transform is needed to be able to correct translation and scale. Registration methods are divided into intensity-based and feature-based methods.(Szeliski, 2006) Feature-based methods consist of four fundamental steps:

\section{Feature detection}

Specialized algorithms detect prominent features like corners in both reference and current channel. Commonly point-based features are determined from descriptors like SIFT (Lowe, 2004), SURF (Bay et al., 2006), ORB(Rublee et al., 2011) which are invariant to certain image distortions.

\section{Feature matching}

Beyond Brute-forcce feature matchers there are a number of fast algorithms available from which the library FLANN (Fast Approximate Nearest Neighbors) is able to choose an appropriate one. (Muja and Lowe, 2009)

3. Parameter estimation

In order to avoid the influence of outliers in the approximation of the parameters the RANSAC algorithms is performed.

With intensity-based methods the whole image is taken into account using different similarity measures. The phase-correlation function (PCF) is for example a fast intensity-based method in Fourier-space using the Fast Fourier Transform (FFT). It calculates the inverse Fourier transformed cross-power spectrum and searches for the peak location which can be extended to perform sub-pixel accuracy by interpolation.(Alba et al., 2012 Foroosh et al., 2002) The motion model estimated by this method is only able to find translation parameters which is insufficient.

Another intensity-based alignment method presented in (Evangelidis and Psarakis, 2008, ?) is maximising the enhanced correlation-coefficient (ECC) as similarity measure. They developed a iterative scheme based on the gradient descent method where the non-linear objective function is approximated by a simpler expression. The great advantage of this similarity measure for the alignment of differing spectral channels is that it is invariant to the occurring photometric distortions.

Figure 9 (upper image) shows an image region of an aligned panorama by PCF where the three most distant spectral columns where merged to a RGB image. The lack of the scale parameter is clearly visible by the colour fringe at edges. As a feature-based method ORB features where used. While its alignment results shown in Figure 9 (middle image) are still not perfect due to the scene dependence, the ECC maximization method in Figure 9 (lower image) shows the best results.

\section{EVALUATION}

Figure 10 shows the final stitched and aligned full $360^{\circ}$ hyperspectral cylindrical panorama with three chosen spectral channels which where merged to RGB from a roof of German Aerospace Center (DLR) in Berlin.

Obvious errors in the resulting panoramic image are due to object movement like clouds, driving cars, flags and changing illumination conditions during acquisition. A higher acquisition rate could minimise these effects resulting also in lower dynamic range. 

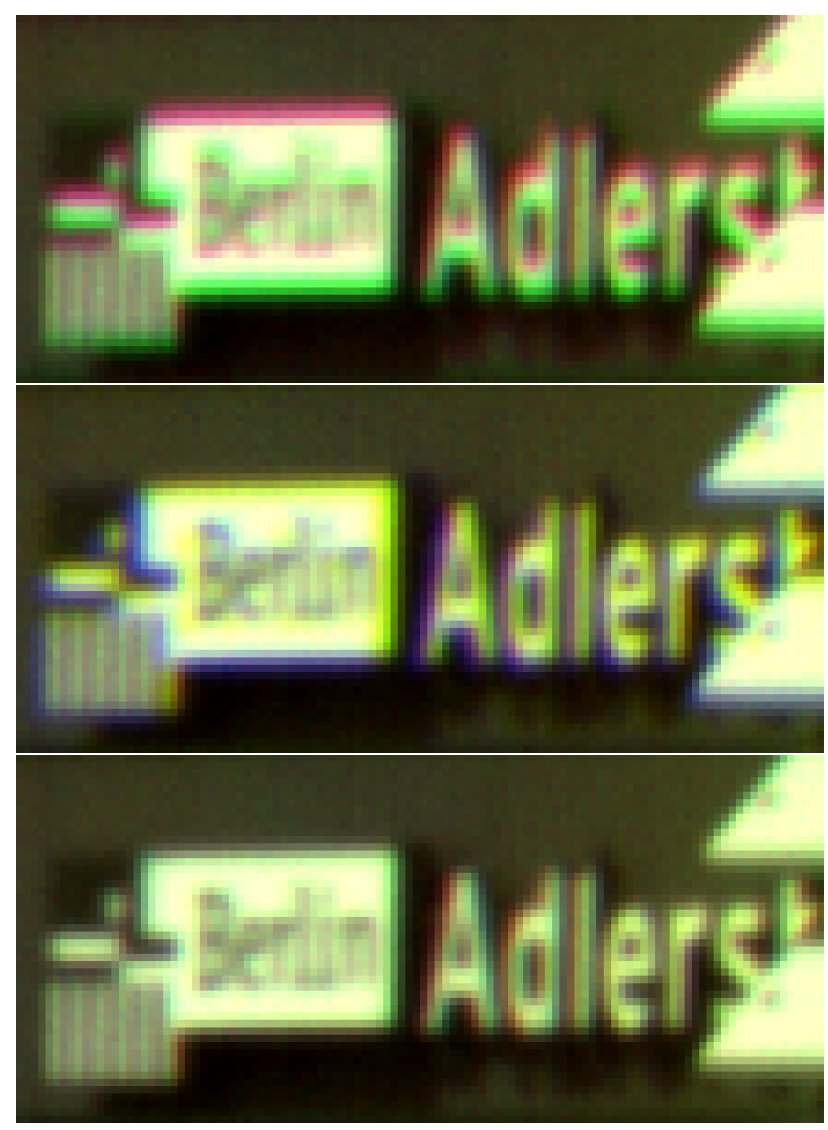

Figure 9. Channel alignment using phase correlation (upper image), matching with ORB features (middle image), enhanced correlation coefficient (ECC) optimization (lower image)

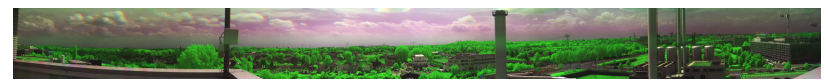

Figure 10. cylinder panorama segment R:977 nm,689 nm $\mathrm{G}: 820 \mathrm{~nm}$ B: $602 \mathrm{~nm}$

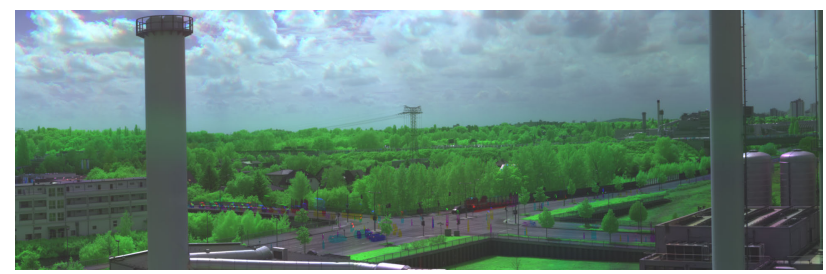

Figure 11. cylinder panorama segment R:977 nm,689 nm $\mathrm{G}: 820 \mathrm{~nm} \mathrm{B:} 602 \mathrm{~nm}$

The spectral information of the captured panorama was evaluated with some common spectral algorithms. An example shows Figure 12 where the normalised differential vegetation index was calculated and a vegetation classification using a threshold was performed.

The interactive visualisation and analysis framework for multiand hyperspectral data Gerbil (Jordan et al., 2016) is an OpenSource software which implements, among other things, some false-colouring algorithms which map the spectral information to RGB values using dimensionality reduction. Figure 13 shows the result of false-coloring using a self-organizing map (SOM) as described in (Jordan and Angelopoulou, 2013).

A SOM is an artificial neural network (ANN) which is trained in
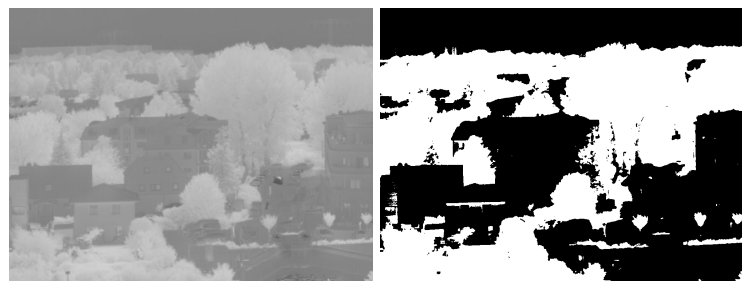

Figure 12. NDVI of an image ROI

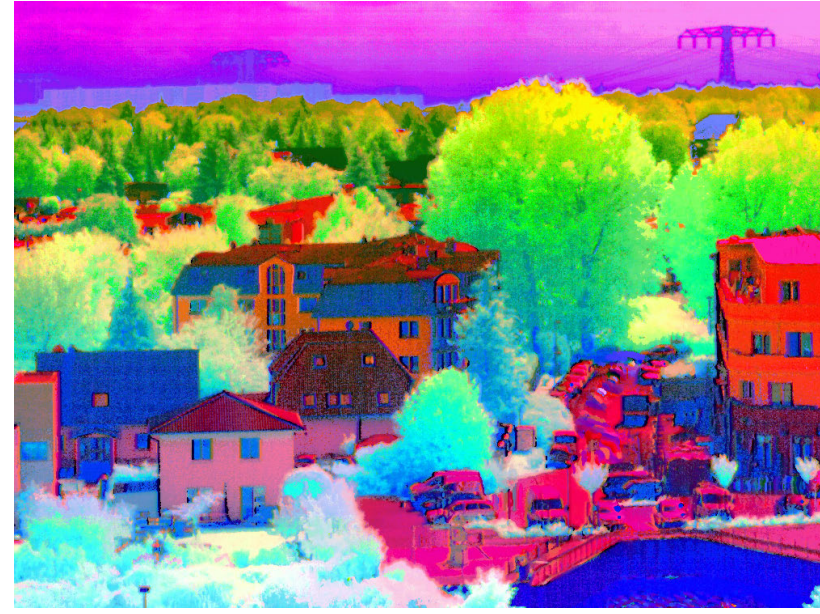

Figure 13. False-coloring of an image ROI with a self organizing map (SOM) using the spectral gradient feature

an unsupervised way. It learns non-linear statistic relations from high dimensional data to transform them to a much simpler net topology. The coordinates of the neurons are then mapped to RGB values. As input we selected the spectral gradient material descriptor presented in (Angelopoulou et al., 1999). The results show that the objects of the scene are distinguishable by their spectrum.

\section{CONCLUSION AND OUTLOOK}

In this work, a scanning hyperspectral panoramic imaging system was presented. It is a low-cost hyperspectral scanning device analogous to the push broom scanner. It has a 2D detector with variable spectral filters, with each filter positioned perpendicular to the direction of flight.

As a result, a long image stripe is created for each spectral channel. These are offset according to their position on the 2D chip and must be co-registered pixel by pixel. For this purpose, different methods were investigated. Further challenges are the data acquisition and the complex corrections of the digital data.

The application of spectral algorithms showed that the detector performs well enough, despite of its spectral properties and second order harmonics. The acquisition of a hyperspectral cylindrical panorama was successfully shown. It is possible to test this in further applications like medical imaging or under more complicated conditions for example in aerial photography.

In addition, an example showing the advantages of a possible application is explained.

\section{REFERENCES}

Alba, A., Aguilar-Ponce, R. M., Vigueras-Gómez, J. F. and ArceSantana, E., 2012. Phase correlation based image alignment with 
subpixel accuracy. In: Mexican International Conference on Artificial Intelligence, Springer, pp. 171-182.

Angelopoulou, E., Lee, S. W. and Bajcsy, R., 1999. Spectral gradient: a material descriptor invariant to geometry and incident illumination. In: Computer Vision, 1999. The Proceedings of the Seventh IEEE International Conference on, Vol. 2, IEEE, pp. 861-867.

Bay, H., Tuytelaars, T. and Van Gool, L., 2006. Surf: Speeded up robust features. Computer vision-ECCV 2006 pp. 404-417.

Evangelidis, G. D. and Psarakis, E. Z., 2008. Parametric image alignment using enhanced correlation coefficient maximization. IEEE Transactions on Pattern Analysis and Machine Intelligence 30(10), pp. 1858-1865.

Foroosh, H., Zerubia, J. B. and Berthod, M., 2002. Extension of phase correlation to subpixel registration. IEEE transactions on image processing 11(3), pp. 188-200.

Hamblenne, A., 2004. The grid - method for a precise location of the entrance pupil on a dslr camera. Outline Graphic Designer.

Huang, F., Klette, R. and Scheibe, K., 2008. Panoramic imaging: sensor-line cameras and laser range-finders. Vol. 11, John Wiley $\&$ Sons.

Huang, F., Wei, S. K. and Klette, R., 2006. Rotating line cameras: model and calibration.

Imec, Jerome Baron, 2017. Linescan hyperspectral image sensor. https://www.imec-int.com/ drupal/sites/default/files/inline-files/

LS100linescanhyperspectralimagesensor_0.pdf last visited: $16 / 7 / 2018$.

Jordan, J. and Angelopoulou, E., 2013. Hyperspectral image visualization with a 3-d self-organizing map. In: Proceedings of the Workshop on Hyperspectral Image and Signal Processing: Evolution in Remote Sensing (WHISPERS13), pp. 1-4.

Jordan, J., Angelopoulou, E. and Maier, A., 2016. A novel framework for interactive visualization and analysis of hyperspectral image data. Journal of Electrical and Computer Engineering 2016, pp. 2.

Karaca, A. C., Ertürk, A., Güllü, M. K. and Ertürk, S., 2016. Ground-based panoramic stereo hyperspectral imaging system with multiband stereo matching. IEEE Journal of Selected Topics in Applied Earth Observations and Remote Sensing 9(9), pp. 3926-3940.

Lowe, D. G., 2004. Distinctive image features from scaleinvariant keypoints. International journal of computer vision 60(2), pp. 91-110.

Mansouri, A., Marzani, F. and Gouton, P., 2005. Development of a protocol for ccd calibration: application to a multispectral imaging system. International Journal of Robotics and Automation 20(2), pp. 94-100.

Muja, M. and Lowe, D. G., 2009. Fast approximate nearest neighbors with automatic algorithm configuration. VISAPP (1) 2(331340), pp. 2.

Rublee, E., Rabaud, V., Konolige, K. and Bradski, G., 2011. Orb: An efficient alternative to sift or surf. In: Computer Vision (ICCV), 2011 IEEE international conference on, IEEE, pp. 25642571.

Spiess, C. and Reulke, R., 2017. Vergleich der optischen eigenschaften von hyperspektralen kameras. In: 37. WissenschaftlichTechnische Jahrestagung der DGPF, DGPF.
Szeliski, R., 2006. Image alignment and stitching: A tutorial. Foundations and Trends $\mathrm{\circledR}$ in Computer Graphics and Vision 2(1), pp. 1-104.

Tack, N., Lambrechts, A., Soussan, P. and Haspeslagh, L., 2012. A compact, high-speed, and low-cost hyperspectral imager. In: Proc. SPIE, Vol. 8266, p. 82660Q 